mobility disability. We pilot-tested a combination of groupbased cognitive-behavioral skills training (CBST; 12, 1-hour classes) and community-based exercise (Enhance Fitness [EF]; 1-hour classes, 3 days/week of balance, endurance, and strength training for 4 months). Eighteen adults age $\geq 65$ years with painful knee OA were randomized 2:1 to either CBST+EF or an active control condition of health education (HE) and EF. Class attendance rates and treatment satisfaction ratings were high and similar between treatment groups. The CBST+EF group had large, clinically significant improvements (pre-post treatment change) in pain, physical performance, and accelerometer-measured physical activity (P-values $<0.05)$, while the control group (HE+EF) had minimal-to-moderate improvements in outcomes. Exit interviews indicated that participants valued and benefitted from the combination of CBST with EF, particularly the pain coping skills training and pedometerbased step monitoring and goal-setting.

\section{IMPROVING OUTCOMES FOR OLDER VETERANS WITH CHRONIC BACK PAIN AND DEPRESSION: INTERVENTION DEVELOPMENT}

U.E. Makris ${ }^{1}$, L. Fraenkel, MD, $\mathrm{MPH}^{2}$, A. Naik, MD ${ }^{3}$, M. Cary Reid, MD, $\mathrm{PhD}^{4}, 1$. VA North Texas Health Care System, Dallas, Texas, United States, 2. VA Connecticut Health Care System, West Haven, CT, USA; Yale University, New Haven, CT, USA, 3. MIchael E. DeBakey VA Medical Center, Houston, TX, USA; Baylor College of Medicine, Houston, TX, USA, 4. Weill Cornell Medical College, New York, NY, USA

Chronic low back pain (cLBP) and depression are highly prevalent, disabling, costly, and complicate each other's management. A feasible, effective behavioral intervention targeting cLBP and comorbid depression is needed, especially in older adults. The goal of this research is to develop, in an iterative process, a health coach-telephone-delivered, behavioral intervention that will ultimately improve outcomes of disability and depression in older Veterans with cLBP and depression. This intervention builds on prior literature for chronic pain and mental illness (including goal setting and patient activation) used in older adults. In phase 1 of this research, the intervention content and manual was developed and adapted using input from 14 interviews (via telephone or in-person) with experts (behavioral interventionists, clinical and lifespan developmental psychologists, geriatricians, pain specialists), Veterans and stakeholders (primary care providers). This research has potential to improve patient reported outcomes for medically complex older Veterans with cLBP and comorbid depression.

\section{SESSION 2605 (PAPER)}

\section{NOVEL APPROACHES TO PROVIDER CARE}

\section{ASSOCIATION BETWEEN ENVIRONMENTAL STIMULATION AND APATHY IN NURSING HOME RESIDENTS WITH DEMENTIA}

Y. Jao ${ }^{1}$, W. Liu ${ }^{2}, \mathrm{~K}$. Williams ${ }^{3}$, 1. Pennsylvania State

University, 2. University of Iowa College of Nursing, 3. University of Kansas

Apathy is a prevalent neurobehavioral symptom of dementia. Increasing evidence supports the influence of environments on dementia. Yet, how environments impact apathy is understudied. This study examined the association between environmental stimulation and apathy in nursing home residents with dementia. The study was guided by the NeedDriven Dementia-Compromised Behavior Model, which identifies the link between environments and neurobehavioral symptoms in dementia. This repeated measures study analyzed 104 video observations of staff-resident interactions in 12 nursing homes. Second-by-second behavioral coding using Noldus Observer software was conducted to assess apathy and environmental stimulation, using the PersonEnvironment Apathy Rating scale. The environment subscale includes six items: stimulation clarity, stimulation strength, stimulation specificity, interaction involvement, physical accessibility, and environmental feedback. The apathy subscale includes six items: facial expression, eye contact, physical engagement, purposeful activity, verbal tone, and verbal expression. Multilevel linear models were used for analysis. The video observations included 63 dyads that included 42 staff and 44 residents with moderate to severe dementia. Results showed that apathy was not associated the overall quality of environmental stimulation but was significantly associated with stimulation specificity $(\beta=-2.22, P=.049)$ and environmental feedback ( $\beta=-2.14, \mathrm{P}=.001)$. After controlling for resident age, gender, dementia stage, and functional level, apathy remained associated with environmental feedback $(\beta=-1.59, P=.019)$. Findings reveal that when environmental stimulation is tailored to the individual and prompts engagement, residents show less apathy. This study highlights the importance of environmental stimulation on apathy. Future research should explore interventions that modify environmental stimulation to reduce apathy and improve dementia care.

\section{MOBILIZE RAPID RESEARCH KNOWLEDGE UPTAKE BY USING GAMIFICATION}

\section{Hung ${ }^{1}$, R. Hung 2 , 1. Vancouver General Hospital, 2.}

University of British Columbia

The topic of what motivates staff to accept new research knowledge and apply in practice is an important one to explore. This project involves using gamification (game thinking and mechanics) to support rapid knowledge uptake. The traditional academic publication offers limited effectiveness as practitioners often find the content boring and difficult to retain. This project aimed to increase engagement, accessibility, knowledge, and effectiveness of research knowledge uptake in dementia care among hospital staff. We took a participatory action approach to engage staff to co-design an online game, called the ART \& SCIENCE of Person-Centred Care for learning 10 basic care techniques identified in a dementia research led by the first author. A total of 70 staff members (nurses, physicians, occupational therapist, physiotherapist, and unit clerk) in the medical and mental health programs of Vancouver General Hospital were involved in testing the games by using multiple action cycles. The project was evaluated by a knowledge test and a survey of staff experience. Over 200 staff played the online game to learn dementia care techniques. Staff reported that they not only gained knowledge and skills in caring for patients with dementia but also had fun and enjoyed the competition. The social experience associated with the game stimulated ongoing engagement and active learning among people in the hospital. In this presentation, we will demonstrate 